\title{
Overexpression of platelet-derived growth factor receptor alpha promotes tumor progression and indicates poor prognosis in hepatocellular carcinoma
}

\author{
Tao Wei ${ }^{1, *}, 4$, Li-Na Zhang ${ }^{2,},{ }^{*}$, Yi Lv' ${ }^{1}$, Xiao-Ya Ma ${ }^{2}$, Lei Zhi ${ }^{3}$, Chang Liu ${ }^{1}$, Feng Ma ${ }^{1}$, \\ Xu-Feng Zhang ${ }^{1}$ \\ ${ }^{1}$ Department of Hepatobiliary Surgery and Institute of Advanced Surgical Technology and Engineering, the 1st Affiliated \\ Hospital of Medical College, Xi'an Jiaotong University, Xi'an, Shaanxi Province, 710061, China \\ ${ }^{2}$ Department of Pharmacy, the 2nd Affiliated Hospital of Medical College, Xi'an Jiaotong University, Xi'an, Shaanxi Province, \\ 710004, China \\ ${ }^{3}$ Department of General Surgery, General Hospital of Ningxia Medical College, Yinchuan, Ningxia Hui Autonomous Region, \\ 750004, China \\ ${ }^{4}$ Department of Hepatobiliary and Pancreatic Surgery, The Second Affiliated Hospital, Zhejiang University School of Medicine, \\ Hangzhou, 310009, China \\ "These authors contributed equally to this work \\ Correspondence to: \\ Xu-Feng Zhang, email: xfzhang125@126.com \\ Keywords: Platelet-derived growth factor receptor alpha, Hepatocellular carcinoma, survival, metastasis, CD31 \\ Received: May 09, $2014 \quad$ Accepted: September 29, $2014 \quad$ Published: October 17, 2014
}

\section{ABSTRACT}

Dysregulation of platelet-derived growth factor receptor alpha (PDGFRa) has been documented in various cancers. However, its role in hepatocellular carcinoma (HCC) remains unknown. We and others have examined that upregulation of PDGFRa might be involved in hepatocarcinogenesis. Here, we report that PDGFRa plays a critical role in HCC progression and prognosis. The expression of PDGFRa was markedly higher in human HCC compared to adjacent liver tissues. Although PDGFRA mRNA was decreased in HCC, PDGF-A mRNA was dramatically increased in HCC. Overexpression of PDGFRa was strongly correlated with microvessel density (MVD) of HCC $(p<0.05)$, as well as macroscopic vascular invasion of the tumors $(p<0.05)$. HCC patients with high PDGFRa expression displayed a shorter overall survival and a higher recurrence rate than those with low PDGFRa expression ( $p<0.05$, respectively). Additionally, stable overexpression of PDGFRa in hepatoma cells promoted cell proliferation, migration, invasion and epithelial-mesenchymal transition in vitro. Similarly, an in vivo assay showed that PDGFRa overexpression in hepatoma cells exhibited remarkably tumorigenic potential in tumor size and weight in vivo, which displayed markedly elevated MVD than controls. Thus, our study provided the evidence that PDGFRa may serve as a candidate prognostic marker and a novel therapeutic target for HCC.

\section{INTRODUCTION}

Molecular therapies have gained wide acceptance in tackling malignancies along with accumulated understanding of molecular and cellular mechanisms regulating tumor growth and progression. In particular, interplay between growth factors and cognate receptors is recognized as a major contributor in carcinogenesis, and blocking theses interactions have been introduced with marked efficacy in preclinical models and clinical settings $[1,2]$. Hepatocellular carcinoma (HCC) is commonly known to be notoriously resistant to systematic chemotherapies. But the advent of sorafinib, which is an oral multiple receptor tyrosine kinase inhibitor and exhibits remarkable survival benefit for advanced unresectable HCC [3, 4], brings hope that therapeutic agents could be efficacious in this intractable condition and encourages further development of novel targeted therapeutic approaches.

Platelet-derived growth factor (PDGF) family represents as a prototype of growth factor function. Specifically, four PDGF ligands, namely PDGF-A, $-B$, $-C$ and $-D$, differentially bind with two distinct 
receptor isoforms, PDGF receptor $\alpha(\operatorname{PDGFR} \alpha)$ and $-\beta$, which subsequently activate their downstream signaling cascade and induce various cellular responses $[5,6]$. The molecular pathogenesis mediated via PDGFR $\alpha$ has been sparsely documented in liver development and hepatocarcinogenesis [7-12]. Our previous work revealed that $\beta$-catenin (a well-known oncogene) knockout mice had an unexpected increase of HCC development and the main contributing factor for this paradox was an escaping signaling pathway through PDGFR $\alpha / \mathrm{PI} 3 \mathrm{~K} / \mathrm{Akt}$ [13]. The in vitro studies corroborated that expression of PDGFR $\alpha$ remarkably increased when $\beta$-catenin was inhibited, indicating that PDGFR $\alpha$ and $\beta$-catenin might account for two divergent carcinogenic mechanisms and distinct subgroups of HCC patients [13]. In the current study, we present the evidence that PDGFR $\alpha$ expression promotes

A

A Hepatocelular carcinoma
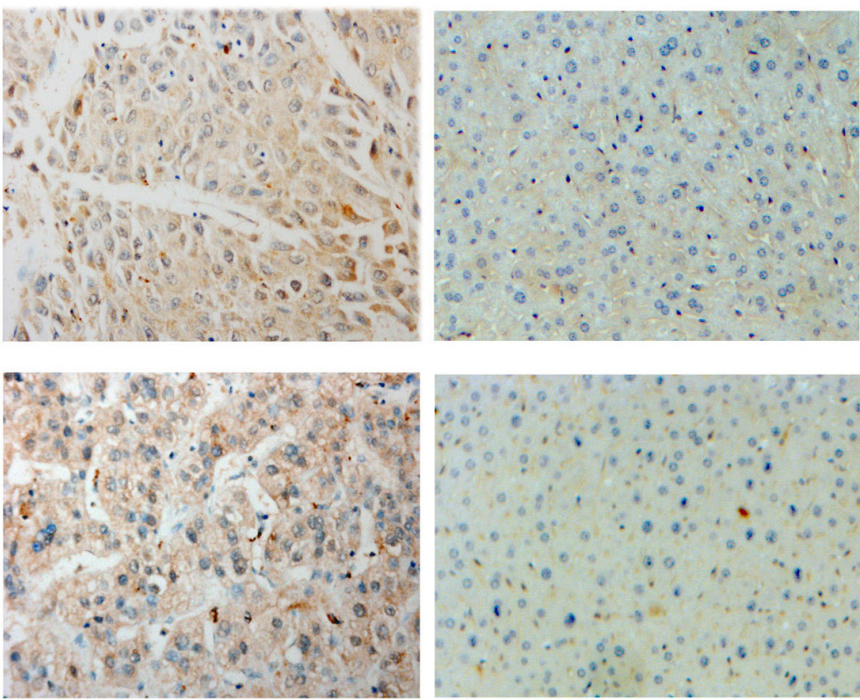

C

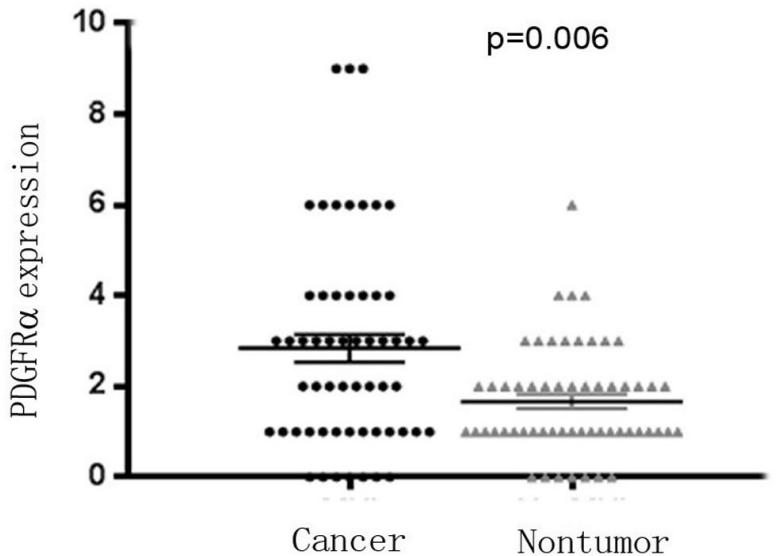

$\mathrm{D}$
HCC growth, invasion and metastasis. Additionally, we show that PDGFR $\alpha$ overexpression predicts poor prognosis in HCC patients undergoing curative resection.

\section{RESULTS}

\section{PDGFR $\alpha$ is upregulated in human HCC tissues}

To explore clinicopathological significance of PDGFR $\alpha$ expression in $\mathrm{HCC}$, we firstly investigated PDGFR $\alpha$ protein expression in 57 pairs of human HCC and adjacent normal liver tissues by immunohistochemistry (IHC) analysis. IHC assays showed that PDGFR $\alpha$ expression was mainly localized to cell membrane and cytoplasm. High protein level of PDGFR $\alpha$ was seen in 22 out of $57(38.6 \%)$ HCC tissues, compared to only 4 out
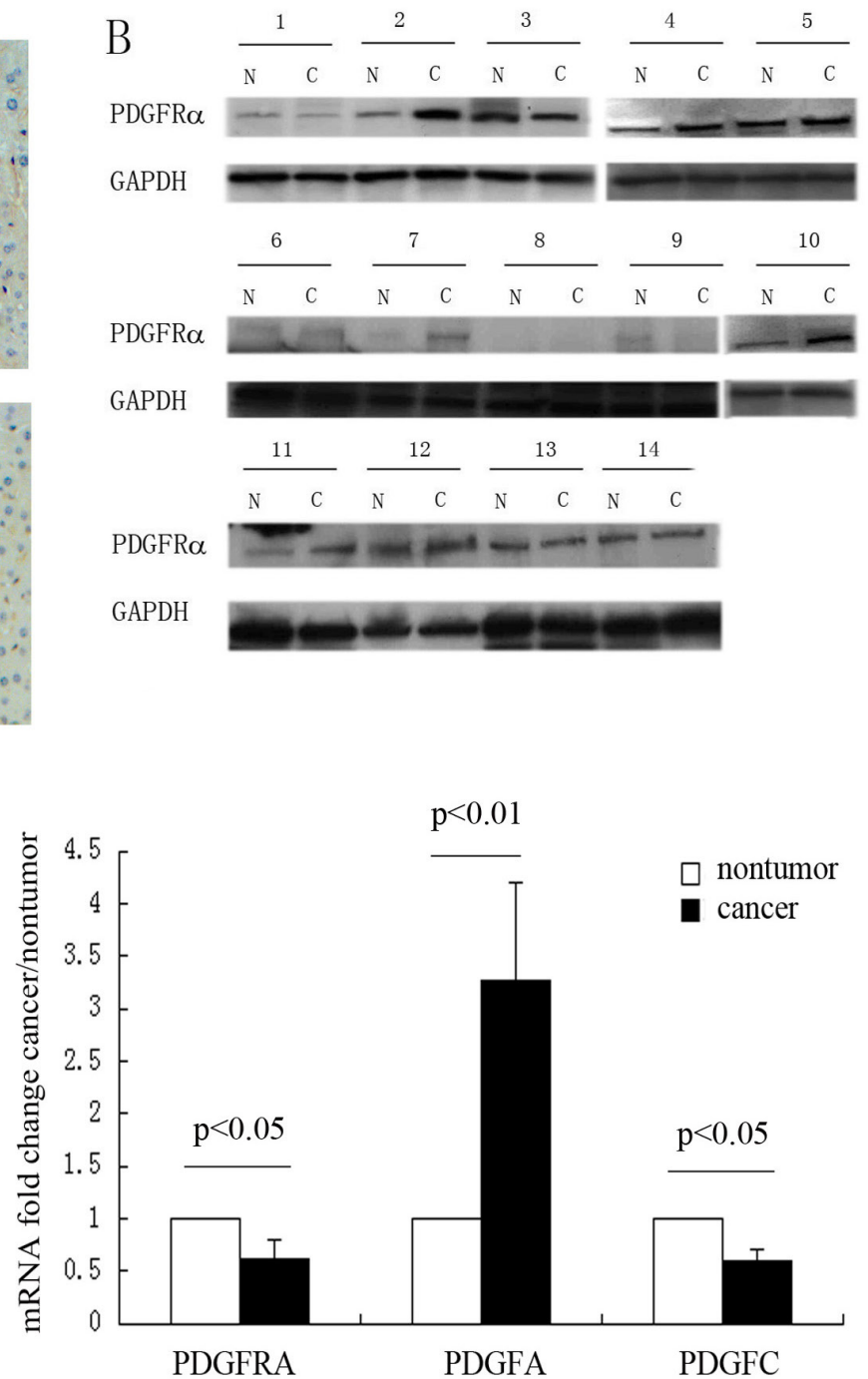

Figure 1: Increased expression of PDGFRa was detected in hepatocellular carcinoma (HCC). (A) Representative IHC staining showed strong PDGFR $\alpha$ expression in human HCC than the adjacent normal liver tissues. Original magnification: 40×. (B) Protein was extracted from the 14 matched HCC and adjacent liver tissues and subjected to western blot analysis to examine different expression levels of PDGFR $\alpha$. (C) Expression level of PDGFR $\alpha$ was scored and compared between HCC and adjacent liver tissues. (D) Comparison of mRNA expression of PDGFR $\alpha$, PDGF-A and PDGF-C between HCC tumor and non-tumor tissues. 
of $57(7.0 \%)$ adjacent liver tissues $(p=0.006)$ (Figure $1 \mathrm{~A}$ and $\mathrm{C}$ ). Additionally, upregulation of PDGFR $\alpha$ protein was confirmed in 14 paired human HCC specimens by western blot analysis (Figure 1B). 5 out of 14 (35.7\%) HCC displayed high PDGFR $\alpha$ protein level compared to the adjacent normal tissues. Intriguingly, the mRNA level of PDGFRA was lower in HCC versus normal liver $(p<0.05)$. To investigate the potential mechanism of PDGFR $\alpha$ upregulation in $\mathrm{HCC}$, we further detected the expression of its ligands, PDGF-A and -C. It was found that PDGF-A but not $\mathrm{C}$ chain was dramatically upregulated in tumors compared to normal liver $(\mathrm{p}<0.01)$. These data indicates that PDGFR ais activated in HCC probably in an autocrine or paracrine fasion.

\section{Correlation of PDGFR $\alpha$ expression and clinicopathological characteristics of $\mathrm{HCC}$}

To further investigate whether high expression of PDGFR $\alpha$ is related to HCC progression, the characteristics of the $57 \mathrm{HCC}$ patients were analyzed. The expression of PDGFR $\alpha$ was assessed by IHC and defined scoring system. The correlation of PDGFR $\alpha$ expression and clinicopathological factors are shown in table 1. Although no significant correlation was observed between PDGFR $\alpha$ expression and liver cirrhosis, tumor size, number, differentiation or alpha-fetoprotein level $(p>0.05)$, statistically significant correlation between PDGFR $\alpha$ expression and macroscopic vascular invasion (MVI) were found $(p=0.010)$. Spearman analysis also revealed that high PDGFR $\alpha$ expression was positively correlated with vascular invasion $(\mathrm{r}=0.212, p<0.001)$.

\section{High PDGFR $\alpha$ expression predicts poor prognosis of HCC after curative surgery}

The survival curves after surgery of HCC patients according to PDGFR $\alpha$ expression were plotted by Kaplan-Meier methods. The results showed patients with high PDGFR $\alpha$ expression had significantly worse overall survival (OS) and disease-free survival (DFS) than those with low PDGFR $\alpha$ expression (log-rank test, $p=0.005$, and $p=0.025$, respectively) (Figure $2 \mathrm{~A}$ and $\mathrm{B}$ ). These findings indicated that HCC patients with high PDGFR $\alpha$ expression had higher risk of tumor recurrence and shorter survival time even after curative surgery.

\section{Analysis of PDGFR $\alpha$ expression in vascular invasion of $\mathrm{HCC}$}

Because the above data identified significant correlation between PDGFR $\alpha$ expression and MVI, we further investigated different PDGFR $\alpha$ expression in HCC with and without MVI. IHC staining identified higher PDGFR $\alpha$

Table 1: Correlation of PDGFRa expression with clinicopathological characteristics of 57 hepatocellular carcinoma specimens

\begin{tabular}{|c|c|c|c|c|}
\hline \multirow[t]{2}{*}{ variables } & \multirow[t]{2}{*}{ NO. } & \multicolumn{2}{|c|}{ PDGFR $\alpha$ expression } & \multirow[t]{2}{*}{$p$ value } \\
\hline & & High $(\%)$ & Low (\%) & \\
\hline $\begin{array}{l}\text { Cirrhosis } \\
\text { Present } \\
\text { Absent }\end{array}$ & $\begin{array}{l}46 \\
11\end{array}$ & $\begin{array}{r}20(43.5) \\
2(18.2)\end{array}$ & $\begin{array}{r}26(56.5) \\
9(81.8)\end{array}$ & 0.122 \\
\hline $\begin{array}{l}\text { Tumor size } \\
\quad<5 \mathrm{~cm} \\
\geq 5 \mathrm{~cm}\end{array}$ & $\begin{array}{l}17 \\
40\end{array}$ & $\begin{array}{r}6(35.3) \\
16(40.0)\end{array}$ & $\begin{array}{l}11(64.7) \\
24(60.0)\end{array}$ & 0.738 \\
\hline $\begin{array}{c}\text { Tumor number } \\
\text { Solitary } \\
\text { Multiple }\end{array}$ & $\begin{array}{r}48 \\
9\end{array}$ & $\begin{array}{r}18(37.5) \\
4(44.4)\end{array}$ & $\begin{array}{r}30(62.5) \\
5(55.6)\end{array}$ & 0.695 \\
\hline $\begin{array}{l}\text { Vascular invasion } \\
\text { Present } \\
\text { Absent }\end{array}$ & $\begin{array}{l}13 \\
44\end{array}$ & $\begin{array}{r}9(69.2) \\
13(29.5)\end{array}$ & $\begin{array}{r}4(30.8) \\
31(70.5)\end{array}$ & 0.010 \\
\hline $\begin{array}{l}\text { Histology } \\
\text { differentiation } \\
\text { Poorly } \\
\text { Moderately } \\
\text { Well } \\
\end{array}$ & $\begin{array}{r}3 \\
37 \\
17\end{array}$ & $\begin{array}{r}0(0) \\
14(37.8) \\
8(47.1)\end{array}$ & $\begin{array}{r}3(100) \\
23(62.2) \\
9(52.9)\end{array}$ & 0.300 \\
\hline $\begin{array}{l}\text { Alpha-fetoprotein } \\
\quad<200 \mathrm{ng} / \mathrm{ml} \\
\geq 200 \mathrm{ng} / \mathrm{ml}\end{array}$ & $\begin{array}{l}12 \\
45\end{array}$ & $\begin{array}{r}2(16.7) \\
20(44.4)\end{array}$ & $\begin{array}{l}10(83.3) \\
25(55.6)\end{array}$ & 0.079 \\
\hline
\end{tabular}


expression in primary HCC with MVI than those without MVI (Figure 3A and B, $p=0.012$ ). Additionally, Patients with MVI had significantly poorer OS than those without MVI

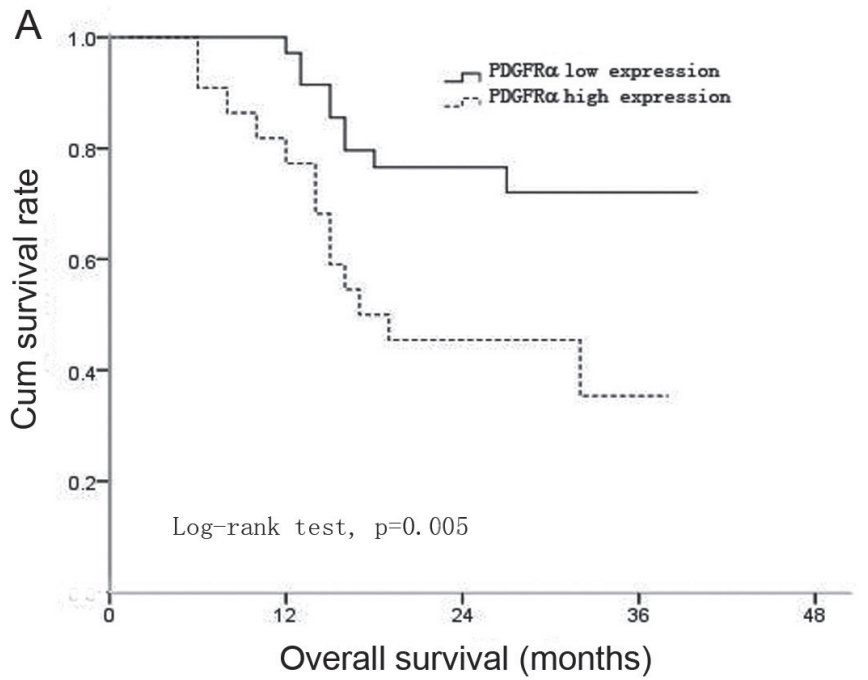

after curative surgery for HCC (Figure 3C, log-rank test, $p<0.001)$. These data suggests that PDGFR $\alpha$ might play a critical role in vascular invasion and metastasis of HCC.

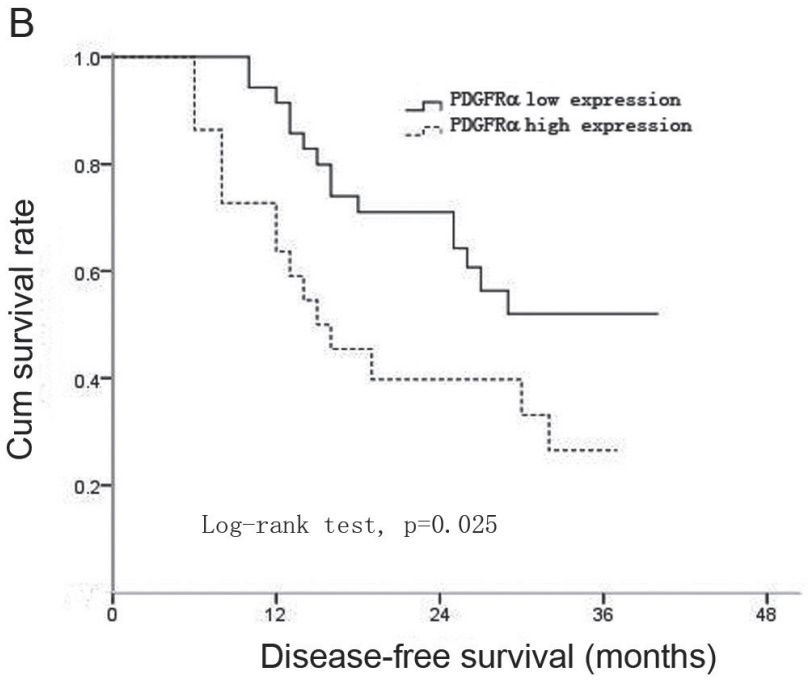

Figure 2: The correlation between PDGFR $\alpha$ expression and overall survival (OS) or disease-free survival (DFS) of the patients after surgery. Curves of OS (A) and DFS (B) were plotted by Kaplan-Meier methods according to PDGFR $\alpha$ expression levels.

A

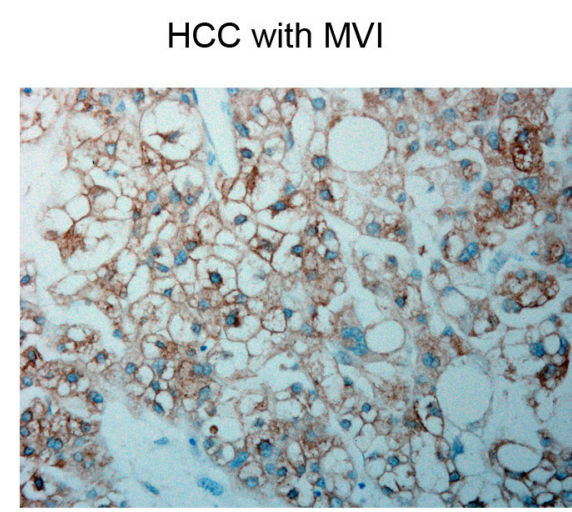

HCC without MVI

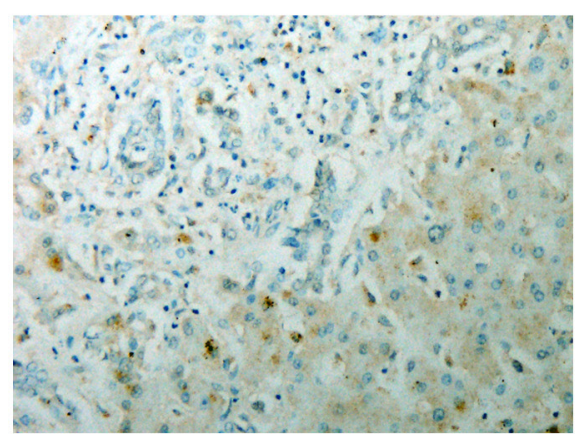

B

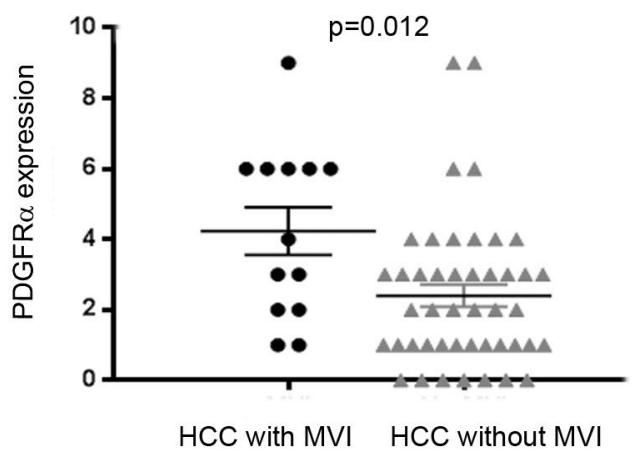

C

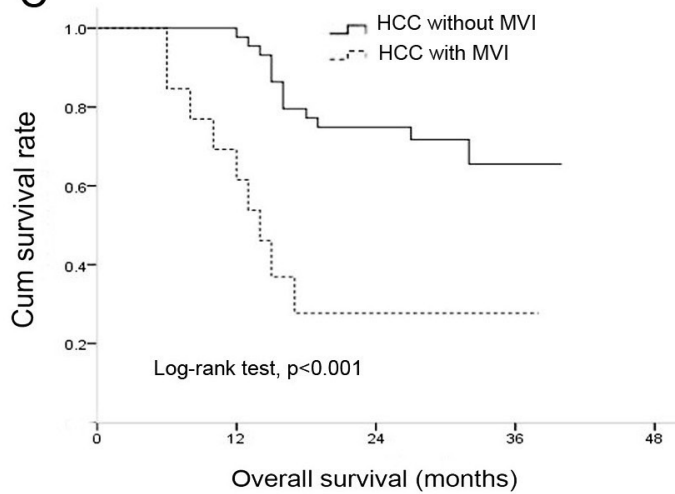

Figure 3: The expression of PDGFRa was analyzed according to macroscopic vascular invasion (MVI) status. (A) Representative IHC staining showed strong PDGFR $\alpha$ expression in primary HCC with MVI than that without MVI. Original magnification: 40×. (B) Quantitative analysis showed higher PDGFR $\alpha$ expression level in HCC with MVI than those without MVI. (C) Kaplan-Meier analysis revealed significantly poorer overall survival in HCC with MVI than those without MVI. 


\section{High PDGFR $\alpha$ expression is positively associated} with increased microvascular density

Next, we examined whether PDGFR $\alpha$ expression was correlated with microvascular density (MVD) in
57 HCC tissues. Specific staining of capillary-like vessels by anti-CD31 was observed in all tumor specimens. In contrast, there is only sparse staining of microvessels in nontumor liver tissues (Fig. 4A). The MVD in 22 patients with high PDGFR $\alpha$ expression $\left(43.3 \pm 4.4 / 0.74 \mathrm{~mm}^{2}\right)$
A

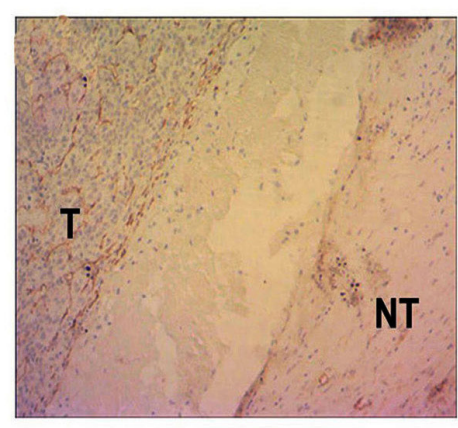

B

PDGFR $\alpha$
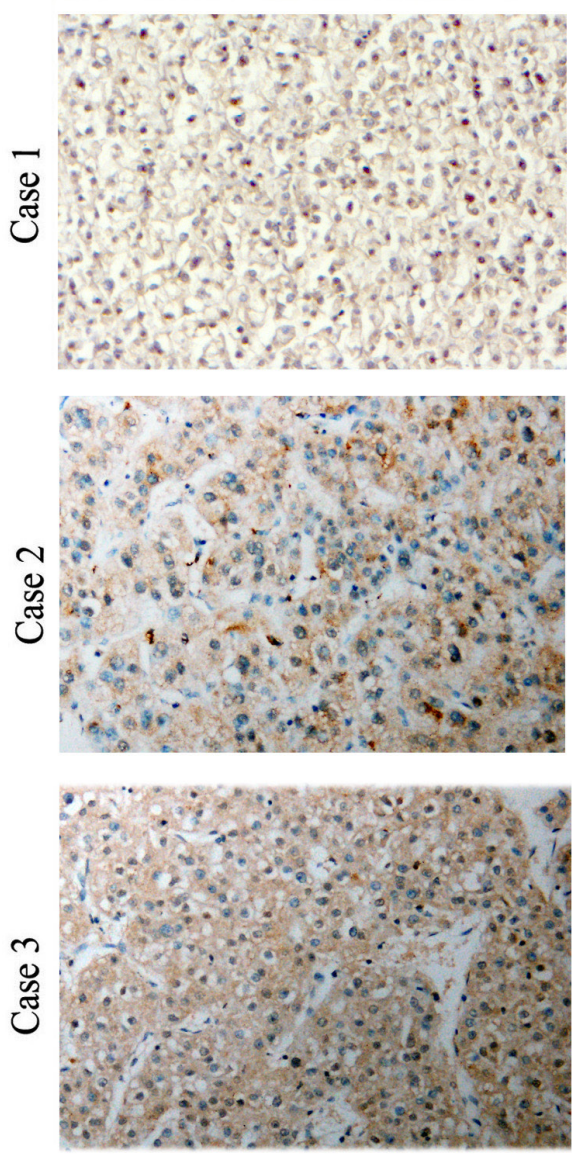

phospho-PDGFR $\alpha$
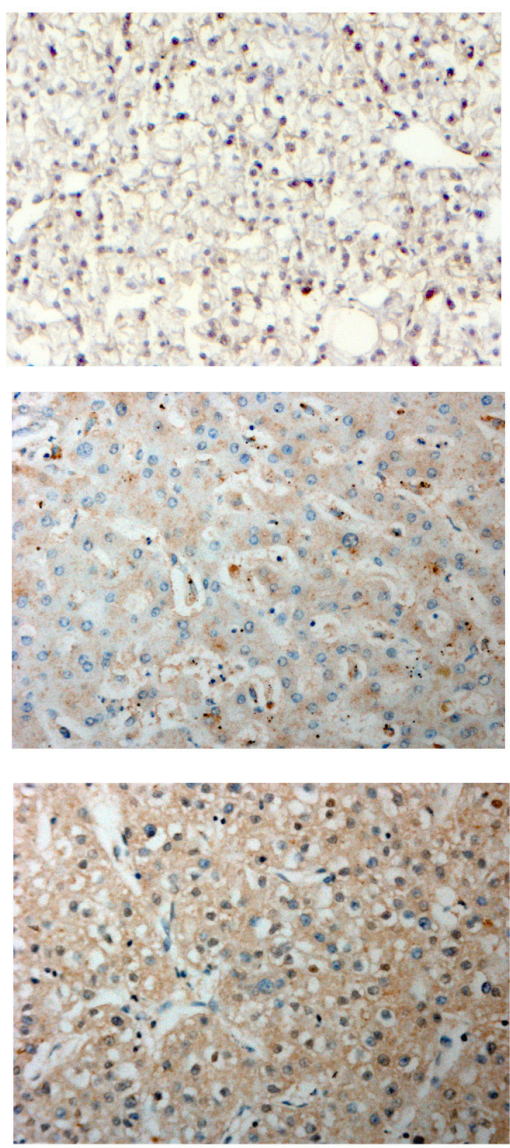

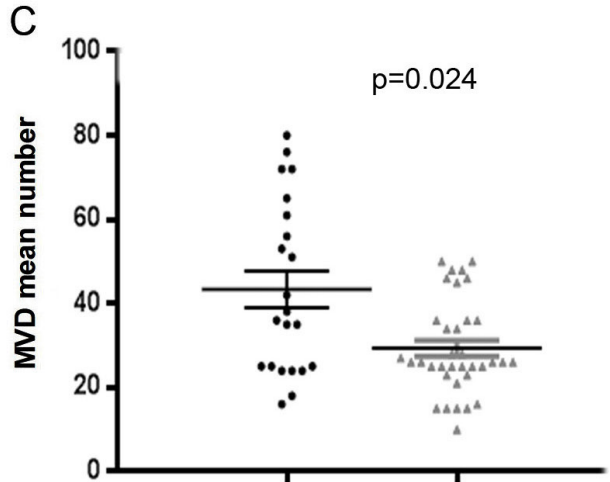

PDGFRa high PDGFRa low

CD31
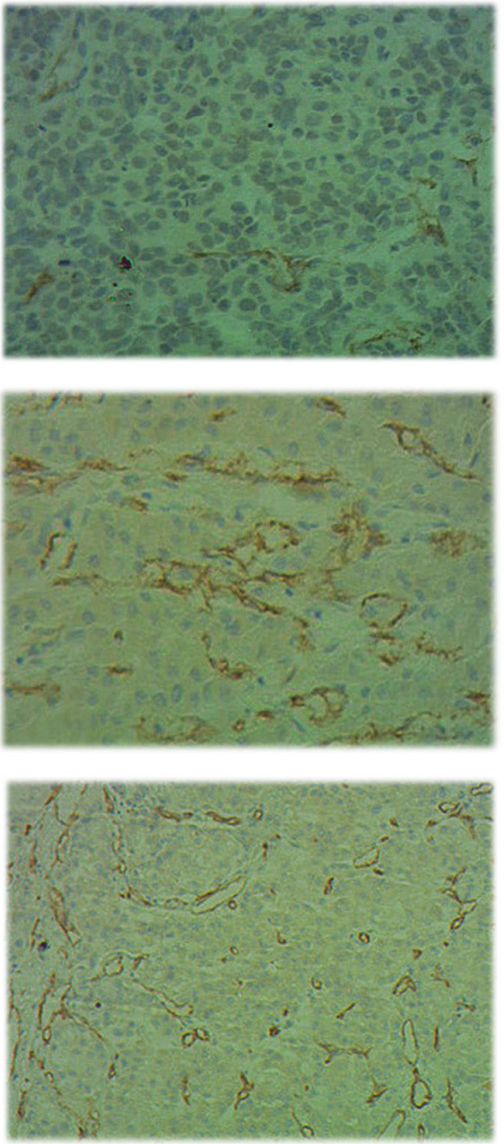

Figure 4: Overexpression of PDGFR $\alpha$ and Tyr ${ }^{754}$-PDGFR $\alpha$ (phosphor- PDGFR $\alpha$ ) in human HCC samples correlated with tumor microvessel density (MVD). (A): Representative IHC staining for CD31 showed remarkable positive staining in HCC than the matched normal liver tissues. Original magnification: $4 \times$ in the left and $40 \times$ in the right panel. (B) Representative IHC staining examined increased MVD in HCC with strong PDGFR $\alpha$ staining than those with weak PDGFR $\alpha$ staining. Original magnification: 40×. (C) Quantitative analysis confirmed correlation of PDGFR $\alpha$ expression level with MVD in HCC. 
was significantly higher than those tumors with low PDGFR $\alpha$ expression $\left(29.2 \pm 1.9 / 0.74 \mathrm{~mm}^{2}\right.$ ) (Fig. 4B and C) $(p=0.024)$. This relationship between PDGFR $\alpha$ expression and MVD indicated that PDGFR $\alpha$ might be a critical factor promoting angiogenesis in $\mathrm{HCC}$.

\section{Stable transfection of PDGFR $\alpha$ in Hep3B cells promotes cell proliferation, migration and invasion}

In vitro experiments were then performed to further address potential impacts of PDGFR $\alpha$ on biological behavior of hepatoma cells. Vectors containing fulllength PDGFR $\alpha$ cDNA were constructed successfully (Suppl. Figure 1). Establishment of stable lentiviral infectants with PDGFR $\alpha$ overexpression (OE) was verified at both mRNA and protein levels (Fig. 5A and B). The thymidine incorporation assay was performed to examine the impact of PDGFR $\alpha$ on cell proliferation. Considering that activation of PDGFR $\alpha$ and downstream signaling requires to be stimulated by its ligands, we added PDGF-AA in cell cultures. Significant increases in thymidine uptake by Hep3B cells with PDGFR $\alpha$ OE, PDGF-AA stimulation and both combined (Figure 5C, all $p<0.05)$ were examined compared to controls. Conversely, treatment of hepatoma cells SK-Hep1 with PDGFR inhibitor Imatinib could significantly decrease cell viability, proliferation or colony formation (Suppl. Figure 2).

Moreover, significant differences were present in both migration and invasion assays between PDGFR $\alpha$ OE and control group (Fig. 5D and E). Hep3B cells are commonly believed to exert very low metastatic potential, and our results also suggested that normal Hep3B scarcely transverse the Matrigel in invasion assay. But remarkably much more cells with PDGFR $\alpha$ OE passed through transwell membrane either or not coated with Matrigel (Fig. 5D and E).

The underlying mechanism for promoted invasion of Hep3B cells after PDGFR $\alpha$ overexpression was further investigated. We found decreased E-cadherin and increased vimentin expression in cells with overexpressing PDGFR $\alpha$, indicating that PDGFR $\alpha$ promoted epithelialmesenchymal transition (EMT) of hepatoma cells (Fig. 5F).

\section{PDGFR $\alpha$ accelerates tumor growth in vivo}

Subsequently, we undertook experiment by injecting PDGFR $\alpha$ OE and control cells into nude mice subcutaneously to assess the role of PDGFR $\alpha$ in tumorigenesis in vivo (Fig. 6A). Tumor xenografts were dissected, the development of tumor lesions was verified by $H \& E$ staining. PDGFR $\alpha$ and subsequent CD31 expression was verified by immunohistochemical staining and western blot (Fig. 6B and C). High PDGFR $\alpha$ expression led to substantially increased tumor volume and tumor weight, as well as increased CD31 expression (Fig. 6B, C, D and E). We further measured the MVD by immunostaining for CD31 in each tissue section, and significantly elevated MVD by 6 to 8 -fold was noticed in the tumor foci of PDGFR $\alpha$ OE group compared with control group (Fig. 6B and F). These findings further indicated that PDGFR $\alpha$ might promote HCC progression partially through enhancing neovascularization.

\section{DISCUSSION}

PDGFR $\alpha$ has been implicated in physiological development of many organs and multiple diseases [14]. It is widely accepted that PDGFR $\alpha$ is activated in various cancers and serves as a potential therapeutic target $[8$, 15, 16]. Among patients with gastrointestinal stromal tumors, seven percent cases harbors activating mutations in PDGFR $\alpha$ and targeted therapy inhibiting PDGFR $\alpha$ significantly prolongs survival of these patients $[17$, 18]. In the present study, we found high expression of PDGFR $\alpha$ protein in a subset of HCC $(\sim 38.6 \%)$ compared to adjacent liver tissues, and concomitant enhanced phospho-PDGFR $\alpha$ immunostaining. However, the mRNA level of PDGFRA was decreased in HCC versus normal liver, indicating possible posttranscriptional modification of mRNA to retard its degradation. Although the initiation mechanism of PDGFR $\alpha$ upregulation remains unknown, increase of its ligand, PDGF-A in HCC might indicate presence of autocrine or paracrine loops in hepatocarcinogenesis [8].

Similar to other oncogenes, remarkably high expression of PDGFR $\alpha$ was observed in specific stages during early liver development but a low level is maintained in normal liver through adulthood [8, 11]. By assuming the commonalities across the organogenesis and tumorigenesis, PDGFR $\alpha$ was found increased in HCC, compared to normal liver in some previous studies $[8,9,19]$. And 3G3, a PDGFR $\alpha$ antibody, could inhibit hepatoma cells growth [8], the mechanism and clinical relevance of which, however, remains unknown. The present study identified that high PDGFR $\alpha$ expression was strongly associated with macroscopic vascular invasion, and thus poor OS and DFS of the patients after surgery. In another study, although a strict grading scale had been used, high PDGFR $\alpha$ in HCC was independently correlated with poor prognosis [9]. These clinical data suggests that PDGFR $\alpha$ contributes to malignant progression of HCC and might be a prognostic marker.

The mechanism of PDGFR $\alpha$ promoting HCC development remains obscure. Intriguingly, a recent study demonstrated that PDGFR $\alpha$ staining predominantly located in small blood vessels instead of tumor cells [10]. Indeed, we also observed positive staining in vessel-like structures, suggesting a role of PDGFR $\alpha$ in regulation of endothelial cells. As firstly identified by the present study, 
A

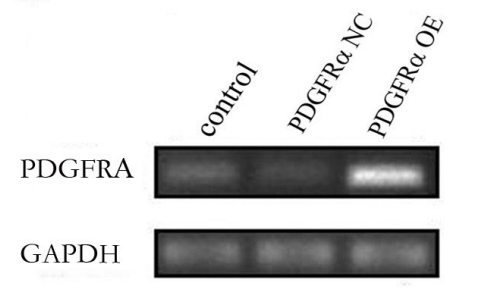

D

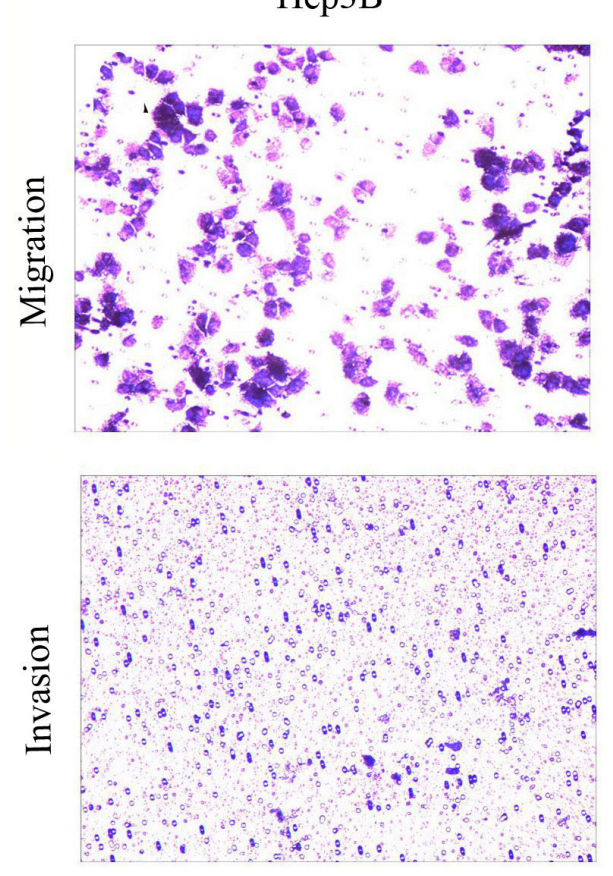

Hep3B

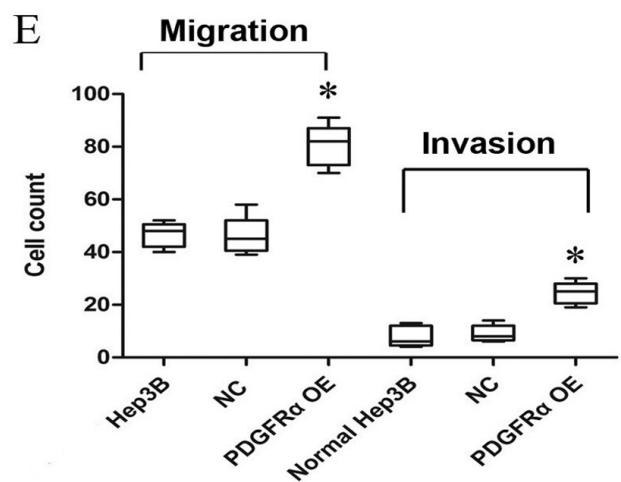

B

PDGFR $\alpha$

$\beta$-actin

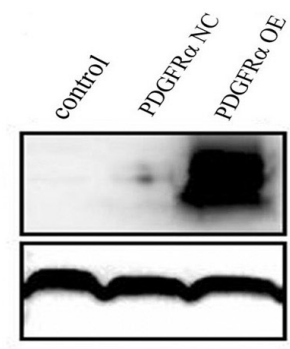

$\mathrm{C}$

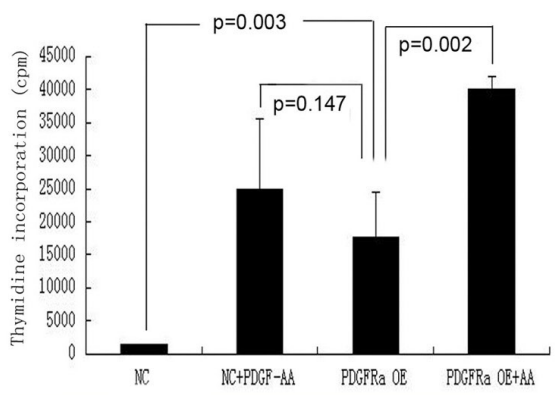

$\mathrm{Hep} 3 \mathrm{~B}+\mathrm{NC}$
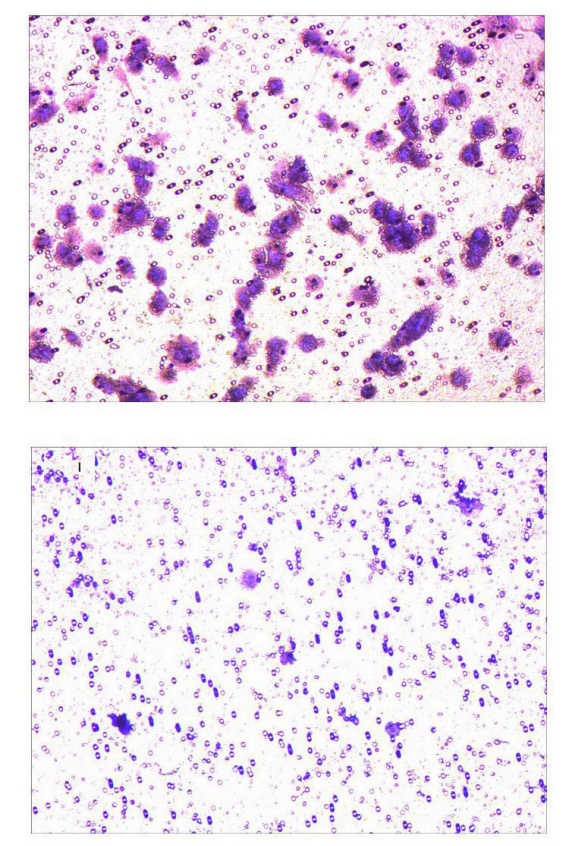

F

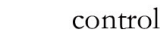

PDGFR

Hep3B+PDGFR $\alpha$ OE
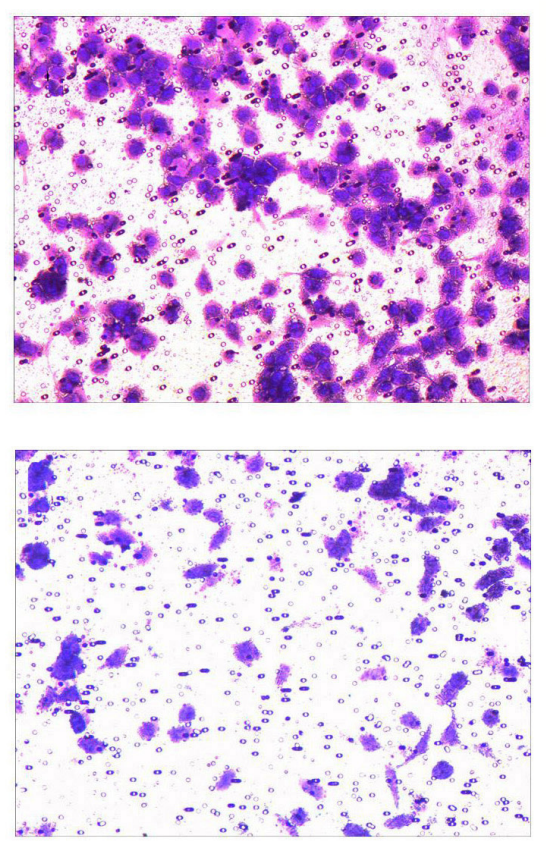

PDGFR NC PDGFR $\alpha$ OE

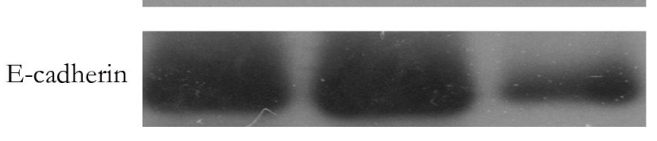

Vimentin

GAPDH
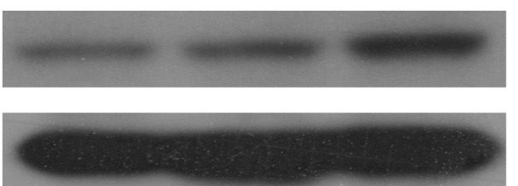

Figure 5: Stable PDGFRa overexpression (OE) in hepatoma cells promoted cell proliferation, migration and invasion. (A \& B): Semiquantitative PCR (A) and immunoblotting (B) analysis of PDGFR $\alpha$ expression levels in Hep3B cells stably transfected by negative control (NC) and PDGFR $\alpha$-lentiviral vector (PDGFR $\alpha$ OE). (C): Cell proliferation was compared by thymidine incorporation assay between cells transfected with PDGFR $\alpha$ NC and PDGFR $\alpha$ OE either with or without addition of PDGF-AA. (D \& E): Cell migration and invasion between cells transfected with PDGFR $\alpha$ NC and PDGFR $\alpha$ OE. (F) Western blot of E-cadherin and vimentin expression between untreated cells and those transfected with PDGFR $\alpha$ NC or PDGFR $\alpha$ OE. $(* p<0.05)$.

both human and mouse HCC tissues with high PDGFR $\alpha$ expression displayed significantly higher MVD than those with low PDGFR $\alpha$ expression, respectively, implying a potential role of PDGFR $\alpha$ in tumor angiogenesis. It is widely established that angiogenesis accounts for a prerequisite for growth and progression of $\mathrm{HCC}$, a typically hypervascular cancer [20]. PDGF possessing similar sequence and structure as vascular endothelial 
A Hep3B-NC
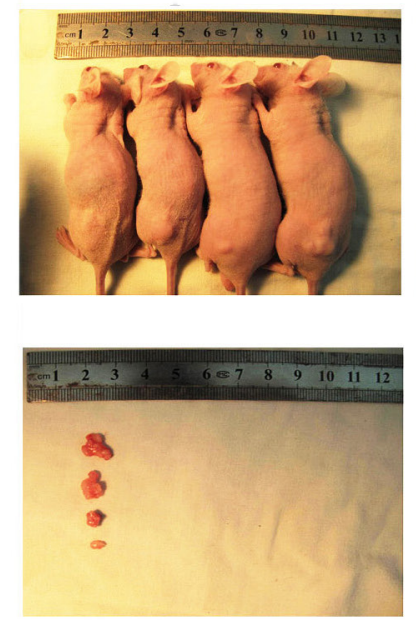

B Hep3B-NC
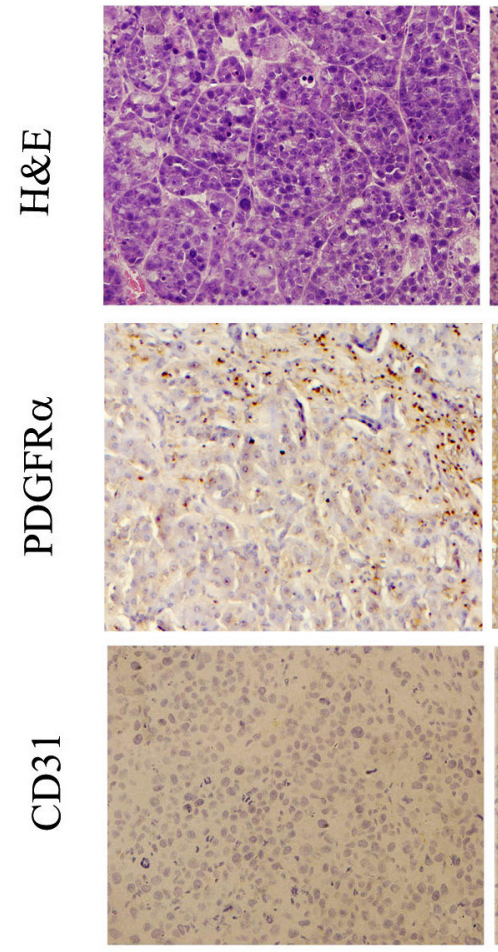

Hep3B PDGFR $\alpha$ OE
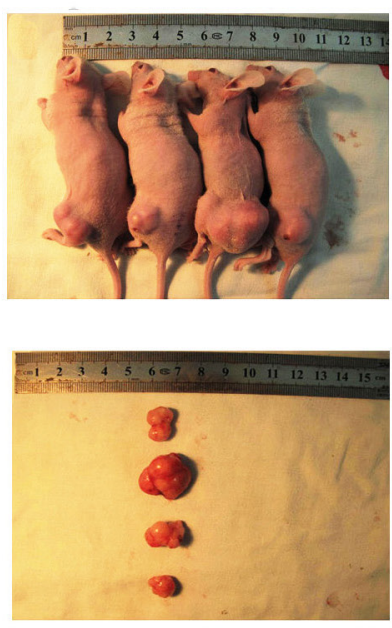

Hep3B PDGFR $\alpha$ OE
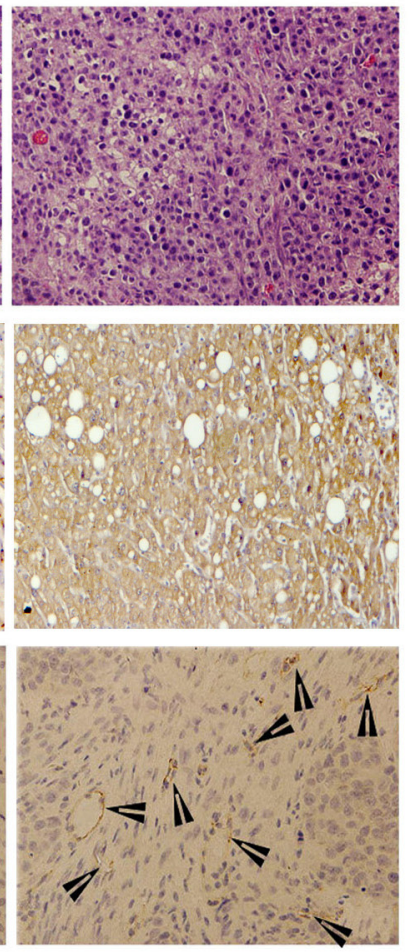

C

NC PDGFR $\alpha$ OE

PDGFR $\alpha$

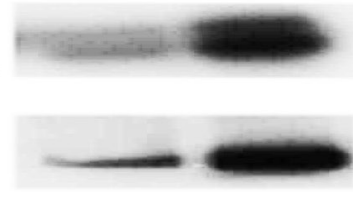

CD31

GAPDH

D

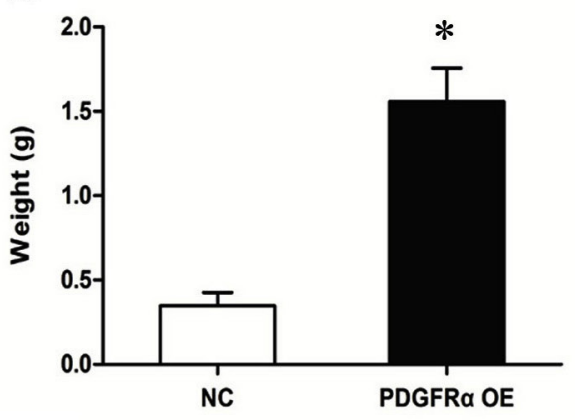

E

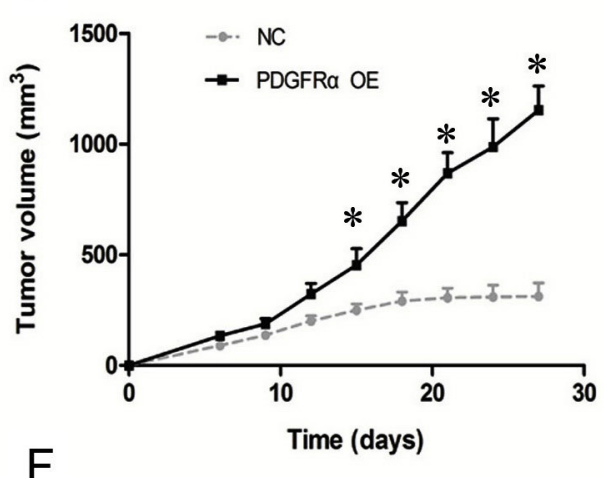

F

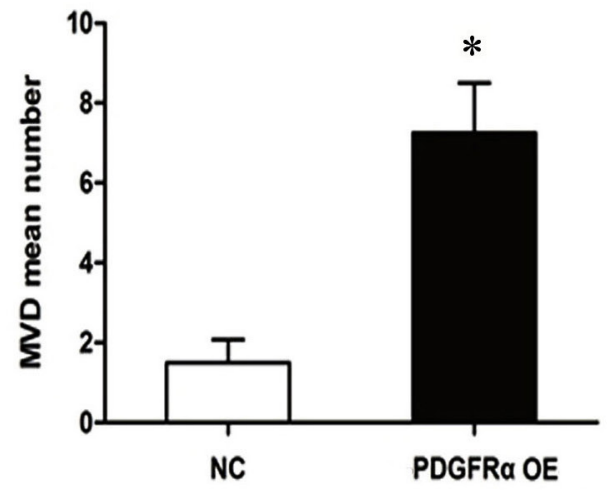

Figure 6: PDGFRa overexpression (OE) promotes tumor growth and progression in vivo. (A): Tumor nodules after subcutaneously inoculation of Hep3B cells transfected with PDGFR $\alpha$ OE or NC for four weeks ( $\mathrm{n}=6$ for each group). (B): H\&E staining and immunostaining for PDGFR $\alpha$ and CD31 of tumors with PDGFR $\alpha$ OE or NC transfection. Original magnification: $20 \times$. The arrowheads indicates vessel-like structures positive for CD31 staining. (C) Immunoblotting for PDGFR $\alpha$ and CD31 of tumors with PDGFR $\alpha$ OE or NC transfection. (D \& E): In vivo tumor growth was compared in tumor weight and volume between cells transfected with PDGFR $\alpha$ OE and NC. (E) Quantitative comparison of MVD between tumors with PDGFR $\alpha$ OE and NC transfection. $(* p<0.05)$. 
growth factor (VEGF) exhibits pro-angiogenic effects in a wide range of conditions [6]. It has been occasionally found that interaction between PDGF and their receptors involves in angiogenesis of liver cancer but not as intensely studied as VEGF [15]. The transgenic mice conditionally overexpressing PDGF-CC in liver spontaneously developed HCC resembling the successive step of human liver tumorigenesis, and activation of endothelial cells was one of the prominent features [21]. And it was further demonstrated in their study that treatment with imatinib led to attenuation of angiogenesis and tumor growth through inhibiting PDGF signaling [22]. Given that PDGF-CC primarily signals through PDGFR $\alpha$, it is rational to speculate the potential influence of PDGFR $\alpha$ on hepatocarcinogenesis, especially on neovascularization.

Several pieces of evidence in this study support a close association of PDGFR $\alpha$ expression and HCC progression. First, PDGFR $\alpha$ expression was significantly higher in HCC with MVI than that in HCC without MVI. Second, HCC with high PDGFR $\alpha$ expression displayed higher MVD than those with low PDGFR $\alpha$ expression. Third, overexpression of PDGFR $\alpha$ in hepatoma cells promotes cell proliferation, migration,invasion and EMT in vitro and tumor growth in vivo. It was previously suggested that PDGFR $\alpha$ overexpression on microvessels was associated with high metastatic potential in HCC cases [7]. Consistently, PDGFR $\alpha$ upregulation was observed on endothelial cells from highly metastatic tumor xenografts and appeared to have a predictive value for HCC recurrence [7]. This finding was corroborated in another clinical study that PDGFR $\alpha$ overexpression in the endothelial cells of HCC tissues was associated with microvascular invasion and high recurrence rate [10]. Interestingly, our in vitro study showed that PDGFR $\alpha$ overexpression in hepatoma cells instead of endothelial cells also resulted in increased metastatic propensity. Further examination of its mechanism revealed that induction of EMT upon PDGFR $\alpha$ upregulation accounted for the enhanced tumor cells invasion, and thus compromised prognosis of HCC patients, as suggested by a recent study that EMT is involved in HCC recurrence after surgery [23]. PDGF-D, a ligand of PDGFR, has been found inducing chemoresistance of $\mathrm{HCC}$ via promoting EMT phenotype of cancer cells [24].

In conclusion, this study demonstrates that overexpression of PDGFR $\alpha$ in $\mathrm{HCC}$ is an indicator of aggressive behavior of tumors and poor clinical outcomes of patients. Therefore, PDGFR $\alpha$ expression may be a candidate biomarker and therapeutic target of HCC.

\section{MATERIALS AND METHODS}

\section{HCC specimens, cell lines and chemical reagents}

30 and 27 pairs of surgically resected HCC and adjacent liver tissues were collected from the 1st and the 2nd Affiliated Hospital of Medical College, Xi'an Jiaotong University, respectively. All 57 pairs of samples were formalin-fixed and paraffin embedded for subsequent immunohistochemical examinations. Another 14 pairs of freshly resected specimens of HCC and adjacent normal liver tissues (at least $3 \mathrm{~cm}$ away from the tumor edges) were collected from the 1st Affiliated Hospital above. The tumor and adjacent normal liver tissues were cut into small pieces and frozen in liquid nitrogen, then store in $-80^{\circ} \mathrm{C}$ refrigerator for further protein analysis. The research was approved by the Ethics Committees of Xi'an Jiaotong University and informed consents were obtained from all enrolled patients.

Human liver cell line HL-7702, HCC cell lines Huh7, HepG2, Hep3B, Snu387, Snu449 and SK-Hep1 were purchased from Shanghai Institute for Biological Science, Chinese Academy of Sciences (Shanghai, China). Cells were cultured in high glucose Dulbecco's modified Eagle's medium (DMEM) or Roswell Park Memorial Institute (RPMI) media 1640 (Hyclone, USA) containing $10 \%$ fetal bovine serum (Hyclone, USA) and $1 \%$ penicillin/streptomycin (Hyclone, USA) and were maintained under the circumstance of $37^{\circ} \mathrm{Cand} 5 \% \mathrm{CO}_{2}$ in a humidified chamber.

Human recombinant PDGF-AA was from PeproTech (USA). The white compound was dissolved in $\mathrm{ddH}_{2} \mathrm{O}$ at $20 \mathrm{ug} / \mathrm{ml}$ and was stored at $-80^{\circ} \mathrm{C}$. A concentration of 50ng/ $\mathrm{ml}$ diluted in culture medium was used in the experiment, unless otherwise stated [25].

\section{Establishment of construct, stable infection and clone selection}

Full-length of wild-type PDGFRA cDNA fragment was synthesized by reverse transcriptase-PCR and amplified (Suppl. Figure 1A). GV-166 vector containing a puromycin selection marker was obtained commercially from Gene Chem (Shanghai, China) (Suppl. Figure 1B). Considering the long sequence of full-length PDGFRA may affect the production of lentiviral particles, the GFP was not added to make the length of vector. The fragment described above was then subcloned into GV166 vector at BamHI/AgeI site and positive product was verified by PCR (Suppl. Figure 1C). The primers for PDGFRA (forward: 5'-AATTCCGTGGTGTTGTCG-3'; reverse: 5'-AAGGTCCGCTGGATTGAG-3'). Then the DNA sequencing confirms the generation of positive clone. In addition, 293T cells were transfected by the construct and western blot show the successful expression of infusion gene (Suppl. Figure 1D and E). The lentiviruses were produced by co-transfecting vector containing gene encoding PDGFR $\alpha$ and packaging plasmids into 293T cells using Lipofectamin 2000. The supernants were collected 48 hours after transfection and filtered via a 0.45 -um filter to acquire the lentiviral particles. For negative controls, empty vector construct containing GFP 
was adopted. Hep3B cells were infected by extracted lentiviruses at multiplicity of infection (MOI) of 50 and then selected by $2 \mathrm{ug} / \mathrm{ml}$ puromycin for 4 weeks. The parallel experiment using control lentiviral vector particle was undertaken and the observation of fluorescence within cells indicated successful infection (Suppl. Figure 1F). Expression of exogenous PDGFR $\alpha$ in Hep3B cells was verified by RT-PCR and western blot.

\section{Immunohistochemistry and assessment of microvessel density}

The prepared 5 um thick paraffin serial sections were deparaffinized by xylene, rehydrated and blocked by $3 \%$ hydrogen peroxide. The following primary antibodies were used to incubate sections overnight at room temperature: PDGFR $\alpha$ (1:50, Santa Cruz), Tyr ${ }^{754}$-PDGFR $\alpha$ (phosphor- PDGFRa, 1:50, Santa Cruz), CD31 (Abcam, 1:100). The sections were stained with biotin-conjugated secondary antibodies and tertiary antibodies conjugated to streptavidin peroxidase. Thereafter, 3,3'-diaminobenzidine staining was done and sections were subsequently counterstained with hematoxylin. Finally, all slides were viewed under the microscope. The percentage of positive cells was divided into four grades (percentage scores): $10 \%$ (0), 10-25\% (1), 25-50\% (2), 50-75\% (3). The intensity of staining was divided into four grades (intensity scores): no staining (0), weak staining (1), moderate staining (2) and strong staining (3). PDGFR $\alpha$ staining positivity was determined by the formula: overall score $=$ percentage score $\times$ intensity score. The overall score $\leq 3$ was defined as negative, and $\geq 4$ was defined as positive.

Microvessel density (MVD) of tissue sections were assessed by two independent observers according to methods described before [26]. Microvessels were defined as brown CD31-immunostained tube-like structure or cell cluster. The sections were screened at $40 \times$ field to select five areas with most intense angiogenesis. At high power field $(200 \times)$, the definite number of microvessels per 0.74 $\mathrm{mm}^{2}$ were counted. The mean microvessel number of five selected areas was considered as MVD.

Additional methods are provided in the Supporting Information.

\section{Statistical analysis}

All in vitro experiements were performed at least 3 times and in triplicate for each individual experiment. All quantitative data were presented as mean \pm standard deviation (S.D.) and were analyzed statistically using one-way ANOVA or 2-sample t test. All categorical variables were expressed as percentage, and were analyzed statistically using Pearson's Chi-square test or Fisher's exact test. Survival curves were plotted using KaplanMeier method, and the differences of survival rate were evaluated by the log-rank test. $\mathrm{p}<0.05$ was considered statistically significant. All calculations and analysis were performed using the SPSS statistic software 13.0 (SPSS, Chicago, IL).

\section{ACKNOWLEDGEMENT}

This work has been supported by National Natural Science Foundation of China (NO. 81372582, 81101873).

\section{Conflict of interest}

The authors declare that there is no conflict of interest.

\section{REFERENCE}

1. Xie Q, Su Y, Dykema K, Johnson J, Koeman J, De Giorgi V, Huang A, Schlegel R, Essenburg C, Kang L, Iwaya K, Seki S, Khoo SK, Zhang B, Buonaguro F, Marincola FM, et al. Overexpression of HGF Promotes HBV-Induced Hepatocellular Carcinoma Progression and Is an Effective Indicator for Met-Targeting Therapy. Genes \& cancer. 2013; 4:247-260.

2. Aravalli RN, Steer CJ, Cressman EN. Molecular mechanisms of hepatocellular carcinoma. Hepatology. 2008; 48:2047-2063.

3. Llovet JM, Ricci S, Mazzaferro V, Hilgard P, Gane E, Blanc JF, de Oliveira AC, Santoro A, Raoul JL, Forner A, Schwartz M, Porta C, Zeuzem S, Bolondi L, Greten TF, Galle PR, et al. Sorafenib in advanced hepatocellular carcinoma. N Engl J Med. 2008; 359:378-390.

4. Cheng AL, Kang YK, Chen Z, Tsao CJ, Qin S, Kim JS, Luo R, Feng J, Ye S, Yang TS, Xu J, Sun Y, Liang H, Liu J, Wang J, Tak WY, et al. Efficacy and safety of sorafenib in patients in the Asia-Pacific region with advanced hepatocellular carcinoma: a phase III randomised, double-blind, placebo-controlled trial. Lancet Oncol. 2009; 10:25-34.

5. Fredriksson L, Li H, Eriksson U. The PDGF family: four gene products form five dimeric isoforms. Cytokine Growth Factor Rev. 2004; 15:197-204.

6. Andrae J, Gallini R, Betsholtz C. Role of platelet-derived growth factors in physiology and medicine. Genes Dev. 2008; 22:1276-1312.

7. Zhang T, Sun HC, Xu Y, Zhang KZ, Wang L, Qin LX, Wu WZ, Liu YK, Ye SL, Tang ZY. Overexpression of platelet-derived growth factor receptor alpha in endothelial cells of hepatocellular carcinoma associated with high metastatic potential. Clinical cancer research: an official journal of the American Association for Cancer Research. 2005; 11:8557-8563.

8. Stock P, Monga D, Tan X, Micsenyi A, Loizos N, Monga SP. Platelet-derived growth factor receptor-alpha: a novel therapeutic target in human hepatocellular cancer. Molecular cancer therapeutics. 2007; 6:1932-1941. 
9. Patel SH, Kneuertz PJ, Delgado M, Kooby DA, Staley CA 3rd, El-Rayes BF, Kauh JS, Sarmiento JM, Hanish S, Cohen C, Farris AB 3rd, Maithel SK. Clinically relevant biomarkers to select patients for targeted inhibitor therapy after resection of hepatocellular carcinoma. Annals of surgical oncology. 2011; 18:3384-3390.

10. Zhu K, Pan Q, Zhang X, Kong LQ, Fan J, Dai Z, Wang L, Yang XR, Hu J, Wan JL, Zhao YM, Tao ZH, Chai ZT, Zeng HY, Tang ZY, Sun HC, et al. MiR-146a enhances angiogenic activity of endothelial cells in hepatocellular carcinoma by promoting PDGFRA expression. Carcinogenesis. 2013.

11. Awuah PK, Nejak-Bowen KN, Monga SP. Role and regulation of PDGFRalpha signaling in liver development and regeneration. The American journal of pathology. 2013; 182:1648-1658.

12. Zhu K, Pan Q, Zhang X, Kong LQ, Fan J, Dai Z, Wang L, Yang XR, Hu J, Wan JL, Zhao YM, Tao ZH, Chai ZT, Zeng HY, Tang ZY, Sun HC, et al. MiR-146a enhances angiogenic activity of endothelial cells in hepatocellular carcinoma by promoting PDGFRA expression. Carcinogenesis. 2013; 34:2071-2079.

13. Zhang XF, Tan X, Zeng G, Misse A, Singh S, Kim Y, Klaunig JE, Monga SP. Conditional beta-catenin loss in mice promotes chemical hepatocarcinogenesis: role of oxidative stress and platelet-derived growth factor receptor alpha/phosphoinositide 3-kinase signaling. Hepatology. 2010; 52:954-965.

14. Heldin $\mathrm{CH}$, Westermark B. Mechanism of action and in vivo role of platelet-derived growth factor. Physiol Rev. 1999; 79:1283-1316.

15. Oseini AM, Roberts LR. PDGFRalpha: a new therapeutic target in the treatment of hepatocellular carcinoma?. Expert Opin Ther Targets. 2009; 13:443-454.

16. Faraone D, Aguzzi MS, Toietta G, Facchiano AM, Facchiano F, Magenta A, Martelli F, Truffa S, Cesareo E, Ribatti D, Capogrossi MC, Facchiano A. Platelet-derived growth factor-receptor alpha strongly inhibits melanoma growth in vitro and in vivo. Neoplasia. 2009; 11:732-742.

17. Hirota S, Ohashi A, Nishida T, Isozaki K, Kinoshita K, Shinomura Y, Kitamura Y. Gain-of-function mutations of platelet-derived growth factor receptor alpha gene in gastrointestinal stromal tumors. Gastroenterology. 2003; 125:660-667.

18. Braconi C, Bracci R, Bearzi I, Bianchi F, Costagliola A, Catalani R, Mandolesi A, Ranaldi R, Galizia E, Cascinu S,
Rossi G, Giustini L, Latini L, Valeri N, Cellerino R. KIT and PDGFRalpha mutations in 104 patients with gastrointestinal stromal tumors (GISTs): a populationbased study. Annals of oncology : official journal of the European Society for Medical Oncology / ESMO. 2008; 19: 706-710.

19. Llovet JM, Chen Y, Wurmbach E, Roayaie S, Fiel MI, Schwartz M, Thung SN, Khitrov G, Zhang W, Villanueva A, Battiston C, Mazzaferro V, Bruix J, Waxman S, Friedman SL. A molecular signature to discriminate dysplastic nodules from early hepatocellular carcinoma in HCV cirrhosis. Gastroenterology. 2006; 131:1758-1767.

20. Zhu AX, Duda DG, Sahani DV, Jain RK. HCC and angiogenesis: possible targets and future directions. Nat Rev Clin Oncol. 2011; 8:292-301.

21. Campbell JS, Hughes SD, Gilbertson DG, Palmer TE, Holdren MS, Haran AC, Odell MM, Bauer RL, Ren HP, Haugen HS, Yeh MM, Fausto N. Platelet-derived growth factor $\mathrm{C}$ induces liver fibrosis, steatosis, and hepatocellular carcinoma. Proc Natl Acad Sci U S A. 2005; 102: 3389-3394.

22. Campbell JS, Johnson MM, Bauer RL, Hudkins KL, Gilbertson DG, Riehle KJ, Yeh MM, Alpers CE, Fausto N. Targeting stromal cells for the treatment of platelet-derived growth factor C-induced hepatocellular carcinogenesis. Differentiation. 2007; 75:843-852.

23. Yamada S, Okumura N, Wei L, Fuchs BC, Fujii T, Sugimoto H, Nomoto S, Takeda S, Tanabe KK, Kodera Y. Epithelial to Mesenchymal Transition is Associated with Shorter Disease-Free Survival in Hepatocellular Carcinoma. Annals of surgical oncology. 2014; .

24. Wu Q, Wang R, Yang Q, Hou X, Chen S, Hou Y, Chen C, Yang Y, Miele L, Sarkar FH, Chen Y, Wang Z. Chemoresistance to gemcitabine in hepatoma cells induces epithelial-mesenchymal transition and involves activation of PDGF-D pathway. Oncotarget. 2013; 4:1999-2009.

25. Rosenkranz S, DeMali KA, Gelderloos JA, Bazenet C, Kazlauskas A. Identification of the receptor-associated signaling enzymes that are required for platelet-derived growth factor-AA-dependent chemotaxis and DNA synthesis. J Biol Chem. 1999; 274:28335-28343.

26. Poon RT, Ng IO, Lau C, Yu WC, Yang ZF, Fan ST, Wong J. Tumor microvessel density as a predictor of recurrence after resection of hepatocellular carcinoma: a prospective study. J Clin Oncol. 2002; 20:1775-1785. 